\title{
Desoneração do ICMS no setor da agropecuária: impactos sobre a economia do estado de Mato Grosso do Sul
}

Mayra Batista Bitencourt Fagundes

Programa de Pós-Graduação em Administração da Universidade Federal do Mato Grosso do Sul (Ufms)

Daniela Teixeira Dias

Grupo de Conjuntura Econômica da Universidade Federal do Mato Grosso do Sul (Ufms)

Daniel Massen Frainer

Curso de Ciências Econômicas da Universidade Estadual do Mato Grosso do Sul (Uems)

Cicero Antônio Oliveira Tredę̧ini

Departamento de Economia e Administração da Universidade Federal do Mato Grosso do Sul (Ufms)

Leonardo Francisco Figueiredo Neto

Departamento de Economia e Administração da Universidade Federal do Mato Grosso do Sul (Ufms)

Recebido: 04/03/2014 Versão revisada (entregue): 18/08/2014 Aprovado: 21/08/2014

\begin{abstract}
Resumo
O objetivo geral deste artigo consiste em analisar os impactos da desoneração do ICMS, incidente na agropecuária, sobre o crescimento e desenvolvimento econômico do estado de Mato Grosso do Sul, bem como os seus efeitos sobre a arrecadação tributária. Para tanto, utilizou-se o método quantitativo descrito na composição e análise da receita setorial, acerca de preços e quantidades por meio da Matriz Insumo-Produto do estado. Diante disso, verificou-se que, com a desoneração, há aumentos na produção, emprego, renda e nas relações intersetoriais, porque a agropecuária é um setor-chave para a economia do estado. Em relação à arrecadação tributária ocorre redução. No entanto, o prazo de recuperação tributária é inferior a um ano. Portanto, os resultados mostram que essa política pública tem impactos favoráveis ao crescimento e ao desenvolvimento econômico.
\end{abstract}

Palavras-chave | Crescimento e desenvolvimento econômico; desoneração de ICMS; impostos; Mato Grosso do Sul.

Código JEL | E62; O13; R15. 


\title{
EXEMPTION OF ICMS IN THE AGRICULTURE SECTOR: IMPACTS ON THE ECONOMY OF THE STATE OF MATO GROSSO DO SUL
}

\begin{abstract}
The purpose of this paper is to analyze the impacts of the exemption of ICMS, incident in agriculture and livestock, on the economic growth and development of the State of Mato Grosso do Sul, as well as its effects on the tax revenues. For this, we used the quantitative method described in the composition and analysis of the sectorial revenues, about prices and quantities, by means of the Input-Output Matrix of Mato Grosso do Sul. It was found that because of exemption there are increases in production, employment, income and intersectorial relations, because agriculture and livestock area key sectors for the state economy. Regarding tax revenue there is a reduction. However, the period of tax recovery is less than one year. Therefore, the results show that this policy has positive impacts on economic growth and development.
\end{abstract}

Keywords | Economic growth and development; Exemption of ICMS; Mato Grosso do Sul; Taxes.

JEL-Code | E62; O13; R15.

\section{EXENCIÓN DEL ICMS EN EL SECTOR AGROPECUARIO: IMPACTOS EN LA ECONOMÍA DEL ESTADO DE MATO GROSSO DO SUL}

\section{Resumen}

El propósito de este trabajo es analizar los efectos de la exención del ICMS incidente en la agricultura y la ganadería en el crecimiento y el desarrollo económico del estado de Mato Grosso do Sul, así como sus efectos en los ingresos fiscales. Para ello, hemos utilizado el método cuantitativo descrito en la composición y el análisis de los ingresos del sector acerca de los precios y cantidades por medio de la matriz de insumo-producto del estado. Como resultado, se verificó que con la exención hay aumentos en la producción, empleo, renta y en las relaciones intersectoriales porque el agropecuario es un sector clave para la economía del estado. En relación a la recaudación tributaria ocurre una reducción que observa un plazo de recuperación inferior a un año. Por lo tanto, los resultados muestran que esa política pública tiene impactos favorables al crecimiento y al desarrollo económico.

Palabras-clave | Crecimiento y desarrollo económico; exención de ICMS; impuestos; Mato Grosso do Sul.

Código JEL | E62; O13; R15.

\section{Introdução}

O desenvolvimento do Mato Grosso do Sul é resultado dos processos de transformações históricas, econômicas, sociais e políticas, que evidenciaram a vocação do estado, desde então, ligado à agropecuária (CORRÊEA, 2011). No estado, essa atividade representa mais de $13 \%$ do PIB, ficando somente atrás do setor de serviços, enquanto que no Brasil essa representatividade é de cerca de 5\%. 
A agropecuária, além de ser considerada como setor chave na produção, é também responsável pelo maior dinamismo regional no comércio exterior, a partir das exportações de carnes, grãos e semimanufaturados (IBGE, 2010).

A importância adquirida pela soja, milho e pecuária referentes, principalmente, aos grãos, carne in natura e semimanufaturados, foi impulsionada pela política tributária brasileira com a criação da Lei Kandir, que, em 1996, desonerou o ICMS - Imposto sobre Circulação de Mercadorias e Serviços - sobre as exportações desses produtos, fato que desestimulou a produção de manufaturados (PINAZZA, 2007).

Apesar disso, ainda sobre as atividades agropecuárias estaduais não relacionadas às exportações, o ICMS representa 80\% de todas as arrecadações incidentes (UFMS, 2013), com uma alíquota comum de 12\%, alíquota essa que nos outros estados assume valores diferenciados. Diante da busca por maior competitividade do setor, essa diferenciação acaba desencadeando uma guerra fiscal entre os estados brasileiros (SOUZA; STICCA, 2011).

Com isso, mais recentemente, discute-se a possibilidade de unificação e redução do ICMS para 4\% em todos os estados brasileiros, ao considerar que o peso desse imposto, além de resultar em guerra fiscal, apresenta dupla contagem nos comércios interestaduais, uma na origem e outra no destino, cujo resultado reflete a alta carga tributária evidenciada no custo Brasil (LIMA, 2013).

Nesse sentido, admite-se que a redução da tributação incidente sobre a agropecuária contribui para a competitividade do estado, por tender a reduzir preços e, desse modo, aumentar a quantidade demandada, promovendo o aumento no nível do PIB e de emprego, por meio das relações intersetoriais (PETTI, 1998). Isso porque, de uma forma geral, o planejamento do desenvolvimento e crescimento econômico local buscam manter a base competitiva dos estados, encorajando a produção, a partir de produtos determinados pelo modelo insumo-produto e satisfazendo o fluxo de trabalhadores (BALDWIN, 1979).

Logo, se houvesse uma redução do ICMS, que ainda incide sobre a agropecuária, para 4\%, como proposto pela unificação, quais seriam os impactos sobre o emprego, renda e produto no estado de Mato Grosso do Sul? Assim, tal problema reflete-se no objetivo geral deste artigo, que consiste em analisar os impactos de uma desoneração fiscal sobre o crescimento e desenvolvimento econômico do estado e seus efeitos sobre a arrecadação tributária. Para tanto, utilizou-se de métodos quantitativos, por meio da matriz-insumo-produto de Mato Grosso do Sul, cálculos de preço, quantidade e receita da atividade. "A tabela de insumoproduto descreve o fluxo de bens e serviços entre todos os setores individuais de uma economia nacional durante um determinado período de tempo." (LEONTIEF, 1983, p. 73). Cabe ressaltar que este artigo teve como base o projeto de pesquisa "Construção da Matriz de Insumo-Produto para o estado de 
Mato Grosso do Sul e Análise da Competitividade das Cadeias Produtivas." (UFMS, 2013).

A importância acadêmica está na análise prospectiva, pois se trata de um artigo com enfoque na Matriz insumo-produto, que analisa os impactos da unificação do ICMS na agropecuária sobre o crescimento e desenvolvimento econômico. E ainda, destacando a análise prospectiva, mensura os efeitos desta unificação, antes de efetivamente se consolidar, portanto, contribuindo para o setor público e entidades locais, por oferecer subsídios para a tomada de decisão quanto à eficiência e a eficácia dessa política.

\section{Desenvolvimento local e a matriz insumo produto}

Historicamente, a matriz insumo produto (MIP) originou-se das organizações e formalizações das relações interindustriais realizadas por Leontief (1983), diante da necessidade de sistematização dos dados (FEIJÓ; RAMOS, 2004). Mais contemporaneamente, essa matriz permite também a gestão e o planejamento na tomada de decisões e previsão de resultados futuros (TALAMINI; PEDROZO, 2004).

Isso porque para a promoção do desenvolvimento e crescimento econômico local, o modelo insumo-produto auxilia na determinação dos produtos necessários às principais atividades econômicas, tentando satisfazer o fluxo de trabalhadores e capacitando a economia para conseguir crescimento e desenvolvimento de forma eficiente com base na competividade dos estados (BALDWIN, 1979).

Nesse sentido, entende-se o desenvolvimento local como a eficiência pela busca da maximização da produtividade e geração de satisfação populacional (RODRIGUES, 2000), enquanto que o crescimento local é o resultado do produto, de acordo com as principais atividades econômicas voltadas à geração de produção e renda, diante de uma série de transformações realizadas em cadeia autossustentada (CLEMENTE; HIGACHI, 2000).

As elaborações das matrizes insumo-produto no Brasil iniciaram-se com o Instituto Brasileiro de Geografia e Estatística (IBGE), em 1970, por meio da estruturação das Contas Nacionais voltadas à construção de informações macroeconômicas. Para 2000 e 2005, a MIP formou-se a partir da Tabela de Recursos e Usos (TRU) pelo detalhamento da produção e consumo que determinaram os coeficientes técnicos (IBGE, 2008a).

A MIP parte das identidades da TRU observadas em:

$$
\mathrm{Y}=\mathrm{CI}+\mathrm{y}^{*}
$$

Onde: $\mathrm{Y}=$ Produção; $\mathrm{CI}=$ Consumo Intermediário; e, y' = Valor adicionado. 


\section{$\mathrm{Y}=\mathrm{CI}+\mathrm{CF}-\mathrm{M}$}

Sendo: $\mathrm{Y}=$ Produção; $\mathrm{CI}=$ Consumo Intermediário; $\mathrm{CF}=$ Consumo Final; e, $\mathrm{M}=$ Importações.

$$
y^{\prime}=\Sigma R
$$

Na qual: $\mathrm{y}^{\prime}=$ Valor adicionado; e, $\sum \mathrm{R}=$ Somatória das rendas.

Por meio do consumo intermediário deriva-se a matriz dos coeficientes técnicos de produção (A), que segundo Feijó e Ramos (2010, p. 295) são calculados a partir de:

$$
\mathrm{a}_{\mathrm{ij}}=\mathrm{g}_{\mathrm{ij}} / \mathrm{g}_{\mathrm{j}}
$$

Em que: $\mathrm{a}_{\mathrm{ij}}=$ Coeficiente técnico; $\mathrm{g}_{\mathrm{ij}}=$ Fluxos intersetoriais; $\mathrm{e}, \mathrm{g}_{\mathrm{i}}=$ Produção total.

De modo que a matriz inversa de Leontief (matriz insumo produto) é obtida por:

$$
Z=(I-A)^{-1}
$$

Onde: $\mathrm{I}=$ Matriz identidade; $\mathrm{A}=$ Matriz dos coeficientes técnicos diretos de produção e $Z=$ Matriz de Leontief ou matriz dos coeficientes técnicos diretos e indiretos.

A partir do qual se observam as relações de produto, emprego e renda necessárias para a derivação e análise da matriz por multiplicadores e relações de compra e venda para o consumo intermediário (índices de Rasmussen-Hirchman), relações essas que, de acordo com Souza (2009), são determinantes para o desenvolvimento e crescimento econômico local tendo como base a agropecuária, uma vez que essa atividade possui uma correlação positiva entre o seu crescimento e o crescimento dos demais setores da economia, fenômeno conhecido como efeito multiplicador, e que visa a geração de bem-estar, emprego, renda e produto. Logo o multiplicador de produto do tipo I foi definido por:

$$
o_{j}=\Sigma b_{i j}
$$

Onde: $\mathrm{O}_{j}=$ multiplicador da produção para o setor j; e, bij = elemento da linha i e da coluna $j$ da matriz inversa de Leontief.

O multiplicador do tipo I de emprego:

$$
E_{j}=\sum w_{n+1}, b_{i j}
$$


Em que: $E_{\mathrm{j}}=$ multiplicador de emprego para o setor $\mathrm{j} ; w_{n+1}=$ razão entre pessoas ocupadas no setor e o produto do setor; e, $b_{i j}=$ elemento da linha i e da coluna $j \mathrm{da}$ matriz inversa de Leontief.

Por fim o multiplicador de renda tipo I enfatizado por Pereira (2010) foi:

$$
\begin{gathered}
\mathrm{MR}_{\mathrm{i}}=\frac{\mathrm{GR}_{\mathrm{i}}}{\mathrm{r}_{\mathrm{i}}} \\
\mathrm{R}_{\mathrm{i}}=\mathrm{VA} \mathrm{A}_{\mathrm{ij}} / \mathrm{X}_{\mathrm{i}} \\
\mathrm{GR}_{\mathrm{i}}=\sum \mathrm{b}_{\mathrm{ij}}{ }^{*} \mathrm{r}_{\mathrm{i}} \mathrm{GR}_{\mathrm{i}}
\end{gathered}
$$

Onde: $\mathrm{X}_{\mathrm{i}}=$ renda na produção de uma unidade monetária; $\mathrm{r}_{\mathrm{i}}=$ efeito direto na renda para o setor $\mathrm{i} ; \mathrm{VA}_{\mathrm{ij}}=$ valor adicionado na matriz-insumo-produto; $\mathrm{GR}_{\mathrm{i}}=$ efeito direto e indireto na renda do setor i; e, $\mathrm{MR}_{\mathrm{i}}=$ multiplicador de renda do setor $\mathrm{i}$.

Os índices de ligações de Rasmussen-Hirchman permitem destacar impactos intersetoriais por meio de dois multiplicadores: o de impacto setorial do produto e o efeito do produto nos setores.

$$
\begin{gathered}
U_{\mathrm{j}}=\left(B_{\mathrm{j}} / \mathrm{n}\right) / \mathrm{B}^{*} \\
\mathrm{U}_{\mathrm{i}}=\left(\mathrm{B}_{\mathrm{i}} / \mathrm{n}\right) \mathrm{B}^{*}
\end{gathered}
$$

Onde: $\mathrm{BJ}=$ somatória de todos os elementos da coluna; $\mathrm{B}^{*}=$ média de todos os elementos da matriz; e, Bi = somatória de todos os elementos de uma linha da matriz inversa.

Caso os multiplicadores tenham valores maiores que 1, significam que os setores são dinâmicos e que respondem ou contribuem mais que proporcionalmente ao aumento de uma unidade monetária ou de emprego na economia como um todo; se inferiores, são pouco dinâmicos e respondem menos que proporcionalmente a impactos na economia. Caso $\mathrm{Uj}$ seja maior que 1 , uma mudança da demanda final leva a um incremento do produto total acima da média, para Ui maior que 1, existe um poder de espraiar os efeitos do crescimento (DÜRR; COSTA, 2008).

\section{Guerra fiscal x unificação do ICMS}

Uma das formas de competitividade na agropecuária é pela atratividade dos produtos a preços menores, decorrentes da redução tributária, proveniente principalmente da desoneração do ICMS que recai sobre as exportações - Lei Kandir (BACHA, 2004). Entende-se por ICMS o imposto sobre operações relativas à circulação de mercadorias e sobre prestações de serviços de transporte interestadual, intermunicipal e de comunicação, regulamentado 
constitucionalmente pela Lei Complementar Anterior n ${ }^{\circ} 87$ de 1996 (BRASIL, 1996).

Nas regiões menos desenvolvidas, o ICMS é a principal fonte tributária, ao representar 80\% das arrecadações totais incidentes sobre a agropecuária (UFMS, 2013) e ao manifestar-se como uma das justificativas para barreiras a possíveis desonerações. No entanto, quando se considera a necessidade de atratividade da agropecuária, observa-se que entre os estados brasileiros iniciam-se reduções contínuas desse imposto sem nenhum tipo de regulamento, fato esse que resulta em guerra fiscal (PETTI, 1998).

A fim de solucionar a guerra fiscal foi desencadeada a Medida Provisória no 599 , de 27 de dezembro de 2012, que dispôs compensações pela perda de arrecadação proveniente da redução e unificação da alíquota do ICMS a partir do Fundo de Desenvolvimento Regional (BRASIL, 2012).

A reforma tributária visou a unificação das alíquotas em 4\%, com previsão para a redução interestadual em até oito anos, com um prazo imediato para as regiões sul e sudeste, redução para 7\% em até sete anos no norte, nordeste e centro-oeste. Diante da queda de arrecadação, as estimativas do sistema de compensação atingem 15 bilhões para o nordeste, norte e centro-oeste, desde que a alocação seja automática, a fim de manter as vantagens comparativas e fomentar novos investimentos. Dos 25 bilhões derivados do fundo, 75\% são vinculados aos tributos federais e 25\% administrados pelos estados (BRASIL, 2012).

Esquematicamente, de acordo com a Imagem 1, considerando o ICMS antes da unificação, o ponto de equilíbrio inicial localiza-se com a tributação $\mathrm{P}+\mathrm{t}$, quantidade d e oferta s. Com uma redução do imposto o nível de preços cai para P+t', devido a redução dos custos de produção, aumentando a demanda para d', elevando o produto real para Q' e o nível de emprego para L', enquanto que o produto nominal se desloca sobre a curva $\mathrm{H}$, reduzindo a carga tributária de $\mathrm{A}+\mathrm{E}$ para $\mathrm{B}+\mathrm{F}$ e o peso morto de $\mathrm{B}+\mathrm{F}+\mathrm{G}$ para $\mathrm{G}$. 
Imagem 1 - Representação do impacto de uma desoneração fiscal sobre oferta, preços, produção, emprego e receita tributária

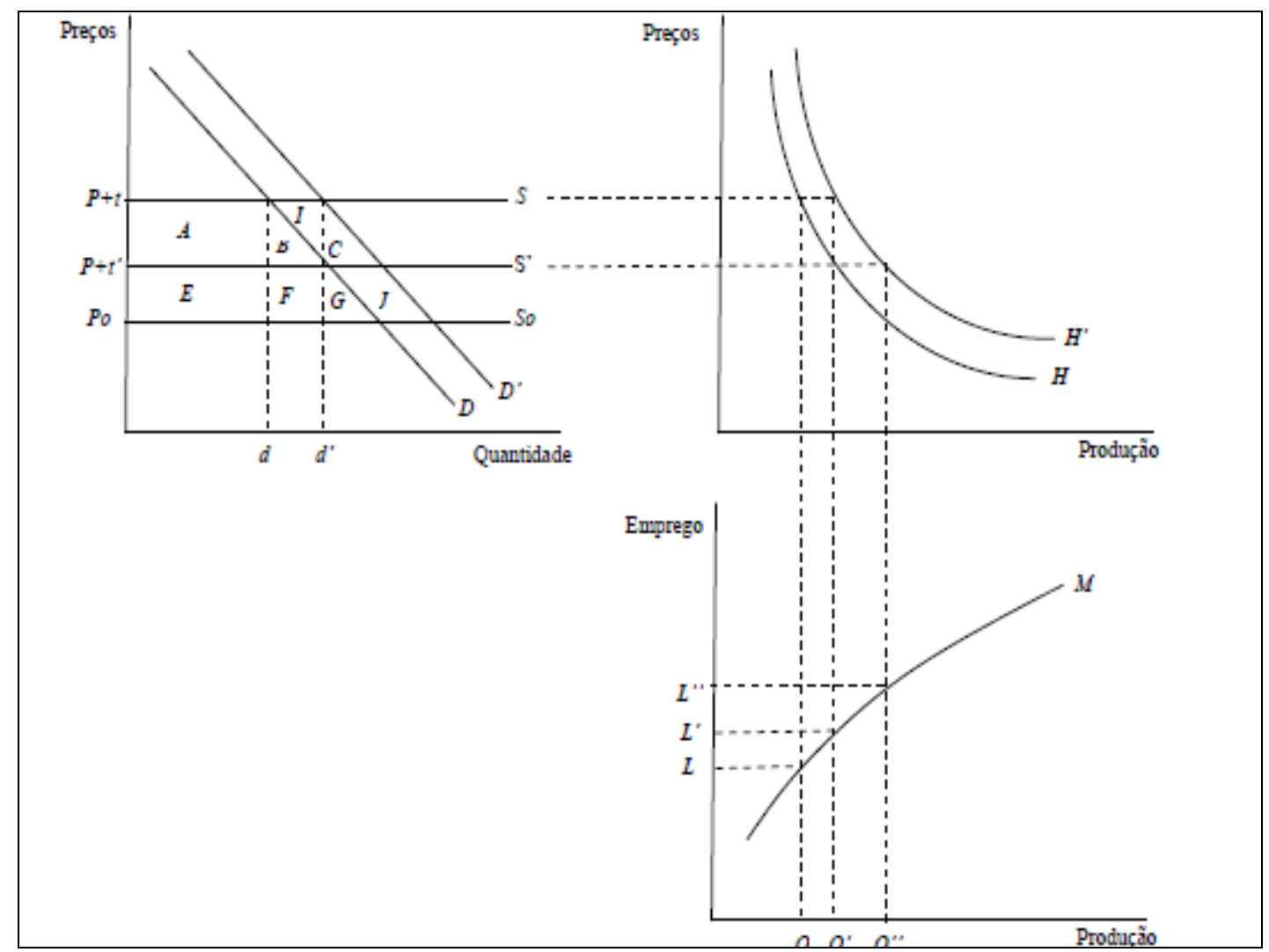

Fonte: Adaptação de Araújo (2007), dos conceitos de Varian (2006), R. Musgrave e P. Musgrave (1980).

\section{Procedimentos metodológicos}

A natureza metodológica é definida por Lakatos e Marconi (2008) como procedimento racional e ordenado, constituído por instrumentos básicos, que implicam utilizar a reflexão e a experimentação de modo a alcançar os objetivos preestabelecidos no planejamento da pesquisa. Nestes aspectos, a natureza da pesquisa foi definida como quantitativa, por salientar o caráter objetivo dos dados numéricos, evitando distorções interpretativas e viabilizando generalizações, teste de hipóteses e validação ou falseamento de teorias por meio da estatística (MAIA NETO; BÊRNI, 2002).

Assim, no primeiro procedimento, as informações partiram da matriz de transações de Guilhoto (2008) com dados para 2004 e dados sobre a agropecuária do IBGE (2010), essenciais para a caracterização da agropecuária no Mato Grosso do Sul, contribuindo, inclusive, na avaliação da importância dessa atividade para os demais setores da economia, enquanto base para a compreensão dos efeitos dos impactos da desoneração sobre esse setor. 
Também se utilizou a composição da matriz insumo-produto regional aberta, ao considerar o Mato Grosso do Sul sem qualquer relação com outra região, e ao enfatizar as relações externas, ao avaliar a importância dessa atividade e como mecanismo para impactos da desoneração fiscal. A MIP foi estimada com base na matriz de transações de 2004 de Guilhoto (2008), constituída por 20 setores, conforme Quadro 1. Cabe destacar que, mesmo sendo o ano base de 2004, os valores relativos são semelhantes da atual realidade.

\section{Quadro 1 - Setores da economia sul matogrossense}

\begin{tabular}{|r|l|c|l|}
\hline $\mathbf{1}$ & Agropecuária & $\mathbf{1 1}$ & Têxtil, vestuário, calçados \\
\hline $\mathbf{2}$ & Mineração & $\mathbf{1 2}$ & Produtos alimentícios \\
\hline $\mathbf{3}$ & Indústria de minerais não metálicos & $\mathbf{1 3}$ & Indústrias diversas \\
\hline $\mathbf{4}$ & Metalurgia & $\mathbf{1 4}$ & Energia elétrica \\
\hline $\mathbf{5}$ & Máquinas e equipamentos & $\mathbf{1 5}$ & $\begin{array}{l}\text { Outros Serviços Industriais de Utilidade } \\
\text { Pública (SIUP) }\end{array}$ \\
\hline $\mathbf{6}$ & Material elétrico e eletrônicos & $\mathbf{1 6}$ & Construção \\
\hline $\mathbf{7}$ & Material de transporte & $\mathbf{1 7}$ & Comércio \\
\hline $\mathbf{8}$ & Madeira, mobiliário, papel & $\mathbf{1 8}$ & Transportes \\
\hline $\mathbf{9}$ & Refino de petróleo e coque & $\mathbf{1 9}$ & Serviços privados \\
\hline $\mathbf{1 0}$ & Outros químicos e farmacêuticos & $\mathbf{2 0}$ & Governo e serviços públicos \\
\hline
\end{tabular}

Fonte: Setores selecionados para análise adaptado de Guilhoto (2008).

Pelo exposto, a partir da matriz de transações mensurou-se a matriz dos coeficientes técnicos pela razão entre o consumo intermediário e valor da produção total de cada setor e produto correspondente. Assim, essa razão que forma uma nova matriz foi deduzida da matriz identidade. A partir do resultado dessa matriz, iniciou o processo de inversão para obtenção da matriz inversa de Leontief, por meio da qual calcularam-se os multiplicadores do tipo I de emprego, renda, produto e os índices de Hasmussem-Hirshman antes e após o impacto da desoneração.

A fim de impactar a matriz insumo-produto elaborada, foi utilizado o modelo de previsão de mercado de projetos de investimento pelas derivações de preços e demandas esperados para a composição da receita esperada, admitindo-se a nova alíquota tributária, descritos por Araújo (2007) em:

$$
\mathrm{Q}_{\mathrm{Q}}=\left[\mathrm{Q}_{0}+\mathrm{Q} \mathrm{ep} \Delta \mathrm{p} / \mathrm{p}\right]\left(1+\mathrm{i}_{\mathrm{q}}\right)
$$


Onde: $\mathrm{Q}_{\mathrm{e}}=$ quantidade demandada no período $\mathrm{n} ; \mathrm{Q}_{\mathrm{e}}=$ quantidade demandada esperada após a redução do imposto; $\mathrm{Q}_{0}=$ demanda atual; $\mathrm{P}_{0}=$ preço atual de mercado; $\mathrm{P}_{\mathrm{e}}=$ preço esperado após a redução do imposto; e, €p = elasticidadepreço da demanda;

Cuja quantidade atual foi estimada a partir de:

$$
\mathrm{R}_{0}=\mathrm{P}_{0} * \mathrm{Q}_{0}
$$

Onde: $\mathrm{R}_{0}=$ receita atual; $\mathrm{P}_{0}=$ preço atual; e, $\mathrm{Q}_{0}=$ quantidade atual.

Considerando o equilíbrio entre produto e demanda, o valor da receita atual refletiu o valor do produto total presente na matriz de transações, descontados os custos de produção e impostos. O preço atual utilizado foi o IPCA disponibilizado pela Uniderp (2004). Com relação à alíquota de imposto atual, o índice foi o comum considerado de 12\% (SOUZA; STICCA, 2011) e a alíquota esperada de 4\% como proposto pela unificação pela Lei $\mathrm{N}^{\circ} 12.512$, de 14 de Outubro de 2011 (BRASIL, 2011).

Sendo:

$$
i_{q}=E_{y} i_{y}
$$

Onde: $\mathrm{i}_{\mathrm{q}}=$ taxa de crescimento do consumo global; Ey = elasticidade-renda da demanda agropecuária; e, $\mathrm{i}_{\mathrm{y}}=$ taxa de crescimento da renda.

A taxa de crescimento da renda considerada foi a taxa de crescimento do PIB (V.A.) agropecuário disponibilizado pela SEMAC/MS e CONAC/IBGE (2010) para o ano de 2004, enquanto que a elasticidade-renda da agropecuária foi a de alimentos de 2004 estimada por Hoffmann (2007).

Considerando-se que são admitidos dois tipos de repasses da desoneração sobre os preços: o repasse total de $100 \%$ e o repasse parcial de $50 \%$, como proposto na desoneração da cesta básica (CRUZ; OMS, 2013), o coeficiente técnico de repasse admitido nesta pesquisa foi de $100 \%$. Assim, a fim de mensurar a redução da carga fiscal tem-se:

$$
\mathrm{P}_{\mathrm{e}}=\mathrm{P}_{0}\left(1+\mathrm{t}_{\mathrm{e}}\right) / 1+\mathrm{t}_{0} \mathrm{cr}
$$

Sendo a receita tributária atual com alíquota de imposto:

$$
\mathrm{R}_{0}=\mathrm{p}_{0} \mathrm{~g}_{0} \mathrm{t}_{0}
$$

Assim, a receita esperada foi estimada por:

$$
R_{\mathrm{a}}=\mathrm{P}_{\mathrm{z}} \mathrm{Q}_{\mathrm{a}} \mathrm{t}_{\mathrm{i}}
$$


Onde: $\mathrm{R}_{\mathrm{e}}=$ receita tributária esperada após a redução do imposto; $\mathrm{R}_{0}=$ receita tributária atual; $\mathrm{t}_{0}=$ alíquota atual de imposto; $\mathrm{t}_{\mathrm{e}}=$ nova alíquota de imposto; $\mathrm{e}$, $\mathrm{Cr}=$ coeficiente de repasse da redução de imposto.

O tempo de retorno foi estimado por:

$$
\mathrm{n}=\ln \left(\mathrm{R}_{0} / \mathrm{R}_{\mathrm{e}}\right) / \mathrm{n}\left(1+\mathrm{i}_{\mathrm{y}} \epsilon_{\mathrm{t})}\right.
$$

$€_{\mathrm{t}}=$ elasticidade-renda do imposto e $\mathrm{i}_{\mathrm{y}}=$ variação percentual da demanda.

A elasticidade preço da demanda foi estimada por Builder [2004]. Sendo a elasticidade tributária calculada pelo método de mínimos quadrados ordinários, no modelo do tipo log-log, para a linearização dos parâmetros, e minimização dos erros (de variáveis fundamentais ocultas e dispersão dos dados), com dados sobre PIB e ICMS provenientes da SEFAZ (2010), em uma série significativa disponível de 2003 a 2010:

$$
\ln \mathrm{T}=\alpha+\beta \ln \mathrm{y}+\mathrm{e}
$$

Onde: $\ln \mathrm{Q}=$ logaritmo de quantidade; lnt $=$ logaritmo de tributo; $\alpha$ e $\beta=$ coeficientes estimados; lny = logaritmo de renda; e, e= erro.

A partir desses resultados assumiram-se novos valores para a agropecuária, valores esses derivados da diferença percentual entre a receita atual e receita esperada, que recaiu positivamente sobre o valor da produção agropecuária, de modo que o aumento do valor da produção foi distribuído de acordo com os coeficientes técnicos na compra e venda do consumo intermediário (a preços básicos - sem impostos) do setor agropecuário, possibilitando o cálculo de novos multiplicadores e índices de Rasmussen-Hirchman, a serem comparados com os efeitos antes do impacto tributário.

\section{Relações intersetoriais da agropecuária}

O desenvolvimento da agropecuária iniciou-se no Mato Grosso do Sul com a divisão do estado de Mato Grosso em 1977, que permitiu a conquista de uma posição geográfica privilegiada, com $25 \%$ de sua área constituída pelo Pantanal e tornou a região uma importante produtora agropecuária (Corrêa, 2011). Diante dessas mudanças, a vocação do Mato Grosso do Sul, desde então, volta-se em direção à agropecuária moderna, com produtividades elevadas em decorrência do solo, clima, água e tecnologia, principalmente de 1977 a 2002, uma vez que essa atividade mantinha-se como a principal do estado (CORR $\hat{E} A, 2011)$.

Assim, a fim de promover o crescimento e o desenvolvimento econômico, programas foram lançados após a divisão do estado, com objetivo de aumentar a produtividade, renda e emprego, tais como: o Programa de Cooperação Nipo- 
Brasileira para o Desenvolvimento dos Cerrados (PROCEDER) como estímulo a agricultura de médio porte; Companhia de Promoção Agrícola (CAMPO); Programa Nacional de Fortalecimento da Agricultura Familiar (PRONAF), que financiava a safra de pequenos agricultores; Programa de Erradicação da Aftosa; além de auxílios de pesquisas da Empresa Brasileira de Pesquisa Agropecuária (EMBRAPA) para o desenvolvimento tecnológico; programas de combate a doenças do Ministério da Agricultura e pelo estímulo proveniente do crédito rural (BRASIL, 2002).

Os resultados dessas propostas de desenvolvimento e crescimento contribuíram para a competitividade. Em 1999 o estado possuía o maior rebanho bovino do país, com uma representatividade de 21,6 milhões de cabeças de gado. Entre 2000 e 2001 atingiu-se a quinta posição na produção de grãos de soja (3 milhões de toneladas), seus cultivos agrícolas representaram $2,8 \mathrm{t} /$ ha nas plantações de soja e 3,1 t/ha nas de algodão, proporcionadores de um crescimento de 3,9 milhões de toneladas para 5,8 milhões dos grãos e caroços entre 1995 e 2002 . Enquanto que a produção de frangos e suínos também foi significativa em 1999 ao representar 17,1 milhões e a de suínos 639 mil (BRASIL, 2002).

De modo que a agropecuária representou 25,2\% do PIB do estado, ao beneficiar 20 mil famílias, com uma disponibilização de recursos de R\$992.000 em 2001. Houve aumento da participação das mulheres e aumento de suas rendas, o que acarretou em melhorias do padrão de vida familiar (BRASIL, 2002). Apesar desses resultados, a partir de 2003 a agropecuária não se comportava mais como principal atividade econômica, mas ainda contribuía para o desenvolvimento e crescimento econômico (IBGE, 2010), considerados complementares e por um desenvolvimento caracterizado pelo avanço, a partir das inovações tecnológicas que tenderam a resultar em aumentos qualitativos e produtivos da agropecuária.

Isso porque a agropecuária possui uma correlação positiva entre o seu crescimento e o crescimento dos demais setores da economia pela geração de bem-estar, emprego, renda e produto (SOUZA, 2009). Nesse sentido, a agropecuária passou a abranger a agricultura temporária e permanente, produção animal, silvicultura e extrativismo vegetal, além disso, foi incorporada também na atividade secundária, a partir da indústria de transformação e, na atividade terciária pelo comércio, transporte e armazenagem (IBGE, 2008b). Com isso, desde 2005, apesar do principal setor econômico concentrar-se nos serviços (IBGE, 2010), a agropecuária assumiu um comportamento ascendente. De modo que os produtos in naturas que mais movimentam o consumo intermediário são descritos ordenadamente por bovinos e suínos, milho, cana-de-açúcar e soja (UFMS, 2013).

Os principais insumos utilizados na produção agrícola (Quadro 2) são descritos, de acordo com a IBGE (2008b), em: adubo, corretivo, aluguel de máquinas, combustíveis, lubrificantes, energia elétrica, sacarias, sementes, mudas, serviços de empreitadas e transporte. Para o cultivo da soja os insumos mais utilizados são 
aproximadamente $30 \%$ provenientes de adubos e corretivos. O milho assemelhase a soja e também utiliza principalmente esses insumos, assim como na cana-deaçúcar. $\mathrm{O}$ diferencial na agricultura manifesta-se com a silvicultura a partir de mais de $32 \%$ advindos dos serviços de empreitadas.

Nesse sentido, observa-se que as principais culturas - tais como a soja, milho e cana-de-açúcar - necessitam da compra de insumos, principalmente de adubos e corretivos, de modo a dinamizar os subsetores responsáveis pela venda de tais insumos.

Quadro 2 - Insumos para a produção agrícola, Mato Grosso do Sul, 2008

\begin{tabular}{|l|r|r|r|r|r|}
\hline & \multicolumn{1}{|c|}{ Soja } & \multicolumn{1}{c|}{ Milho } & $\begin{array}{r}\text { Cana-de- } \\
\text { açúcar }\end{array}$ & Silvicultura & $\begin{array}{r}\text { Outras } \\
\text { culturas }\end{array}$ \\
\hline Adubo e corretivo & $29,31 \%$ & $26,70 \%$ & $29,05 \%$ & $2,37 \%$ & $15,19 \%$ \\
\hline Agrotóxico & $20,77 \%$ & $16,77 \%$ & $8,96 \%$ & $7,81 \%$ & $13,19 \%$ \\
\hline Aluguel de Maq. & $0,99 \%$ & $1,55 \%$ & $2,18 \%$ & $0,26 \%$ & $1,53 \%$ \\
\hline Comb. e lubrificantes & $16,52 \%$ & $18,16 \%$ & $17,66 \%$ & $4,97 \%$ & $15,93 \%$ \\
\hline Energia elétrica & $1,56 \%$ & $2,53 \%$ & $0,60 \%$ & $3,54 \%$ & $6,47 \%$ \\
\hline Sacarias & $0,21 \%$ & $0,69 \%$ & $0,00 \%$ & $0,30 \%$ & $0,60 \%$ \\
\hline Sementes e mudas & $12,87 \%$ & $13,31 \%$ & $6,44 \%$ & $4,39 \%$ & $12,40 \%$ \\
\hline Serv. de empreitadas & $2,23 \%$ & $2,76 \%$ & $9,70 \%$ & $32,97 \%$ & $7,14 \%$ \\
\hline Transporte & $3,20 \%$ & $3,73 \%$ & $7,21 \%$ & $11,03 \%$ & $5,20 \%$ \\
\hline Outros & $12,34 \%$ & $13,81 \%$ & $18,21 \%$ & $28,02 \%$ & $22,34 \%$ \\
\hline
\end{tabular}

Fonte: IBGE (2008b).

No caso da pecuária para a produção de carne bovina (Quadro 3) o insumo mais utilizado é o sal, cerca de $20 \%$. No caso da carne suína e de aves, mais da metade dos insumos deriva da ração industrial. Enquanto que para bubalinos, asinos, muares, coelhos, equinos e caprinos destacam-se ração industrial, sal, combustíveis e lubrificantes. No caso da carne bovina, suína e de aves a ração industrial e sal são utilizados na engorda dos animais destinados ao abate. 
Quadro 3 - Insumos para a produção pecuária, Mato Grosso do Sul, 2008

\begin{tabular}{|l|r|r|r|r|}
\hline & \multicolumn{1}{|c|}{ Bovino } & \multicolumn{1}{c|}{ Suínos } & \multicolumn{1}{c|}{ Aves } & Outros Animais \\
\hline Adubo e corretivo & $9,98 \%$ & $5,56 \%$ & $2,34 \%$ & $10,28 \%$ \\
\hline Agrotóxico & $2,25 \%$ & $3,07 \%$ & $0,93 \%$ & $2,32 \%$ \\
\hline Comb. e lubrificantes & $16,94 \%$ & $7,59 \%$ & $3,26 \%$ & $17,45 \%$ \\
\hline Energia elétrica & $4,60 \%$ & $4,49 \%$ & $2,19 \%$ & $4,74 \%$ \\
\hline Sementes e mudas & $7,26 \%$ & $3,27 \%$ & $1,16 \%$ & $7,47 \%$ \\
\hline Medicamento p/animais & $11,31 \%$ & $4,91 \%$ & $1,64 \%$ & $11,65 \%$ \\
\hline Ração industrial & $2,22 \%$ & $56,36 \%$ & $62,81 \%$ & $18,51 \%$ \\
\hline Sal & $17,98 \%$ & $1,27 \%$ & $0,52 \%$ & $18,51 \%$ \\
\hline Serviço de empreitada & $7,21 \%$ & $2,05 \%$ & $0,92 \%$ & $7,43 \%$ \\
\hline Outros & $20,24 \%$ & $11,42 \%$ & $24,25 \%$ & $17,87 \%$ \\
\hline
\end{tabular}

Fonte: IBGE (2008b).

$\mathrm{Na}$ agricultura o consumo intermediário representa $\mathrm{R} \$ 2.784 .504,66$ (mil) e, na pecuária, R\$ 2.175.979,20 (mil) para a produção preliminar de grãos e carne in natura (IBGE, 2008b). Passado esse processo, a atividade agropecuária tende a vender seus produtos tanto para o consumo intermediário de outras atividades, quanto para o consumo final (GUILHOTO, 2008).

Assim o setor da agropecuária vende para a própria atividade, para o setor de madeira, mobiliário, papel, outros químicos e farmacêuticos, têxtil, vestuário, calçados, produtos alimentícios, serviços privados, governo e serviços públicos, principalmente. A maior parte dessas vendas é destinada ao setor dos produtos alimentícios, cerca de 80\% conforme Quadro 4. 
Quadro 4 - Vendas da agropecuária ao consumo intermediário de outros setores, 2004

\begin{tabular}{|l|c|}
\hline \multicolumn{1}{|c|}{ Setor } & Vendas da agropecuária \\
\hline Agropecuária & $15,04 \%$ \\
\hline Indústria de minerais não metálicos & $0,01 \%$ \\
\hline Madeira, mobiliário, papel & $0,55 \%$ \\
\hline Outros químicos e farmacêuticos & $2,86 \%$ \\
\hline Têxtil, vestuário, calçados & $2,86 \%$ \\
\hline Produtos alimentícios & $80,63 \%$ \\
\hline Indústrias diversas & $0,01 \%$ \\
\hline Comércio & $0,01 \%$ \\
\hline Serviços privados & $0,48 \%$ \\
\hline Governo e serviços públicos & $0,10 \%$ \\
\hline
\end{tabular}

Fonte: Guilhoto (2008).

Internamente, as vendas para outros setores são destinadas as tradings. Caso o destino não seja interno, as vendas serão para consumo final ou para consumo intermediário de outros estados brasileiros e/ou países, nesta distribuição incidem impostos. Assim, 72,72\% da produção agropecuária destinam-se a outras regiões e $27,28 \%$ ao consumo intermediário interno, responsável pela maior dinâmica do estado.

\section{A agropecuária no Mato Grosso do Sul: efeitos multiplicadores sobre o desenvolvimento e crescimento local}

A agropecuária é uma atividade fundamental para a economia estadual e nacional, ao concentrar-se entre as principais atividades relacionadas com o comércio exterior e por impulsionar o setor industrial e de serviços. Consequentemente, vinculando efeitos sobre o desenvolvimento e crescimento local, a partir, principalmente, da geração de produto, emprego e renda (ABIGRAF, 2013; BRASIL, 2002; CONAB, 2005).

Nesse sentido, especificamente, verificou-se por meio dos índices de ligação para frente (a partir do índice de Hasmussem-Hirshman) que a agropecuária é uma atividade que apresenta um dos maiores efeitos sobre a dinâmica da economia local na venda destinada ao consumo intermediário. Verifica-se que o aumento de uma unidade monetária na demanda final leva a um incremento no consumo intermediário e, consequentemente, no produto acima da média entre os demais 
setores. E ainda é mais do que proporcional a essa unidade adicional na economia, em outras palavras, a cada $\mathrm{R} \$ 1,00$ a mais demandado de produto agropecuário, as vendas intermediárias desse setor aumentam em $\mathrm{R} \$ 1,50$, como observado no Gráfico 1.

\section{Gráfico 1 - Índices de ligação para frente antes da desoneração tributária,} 2004

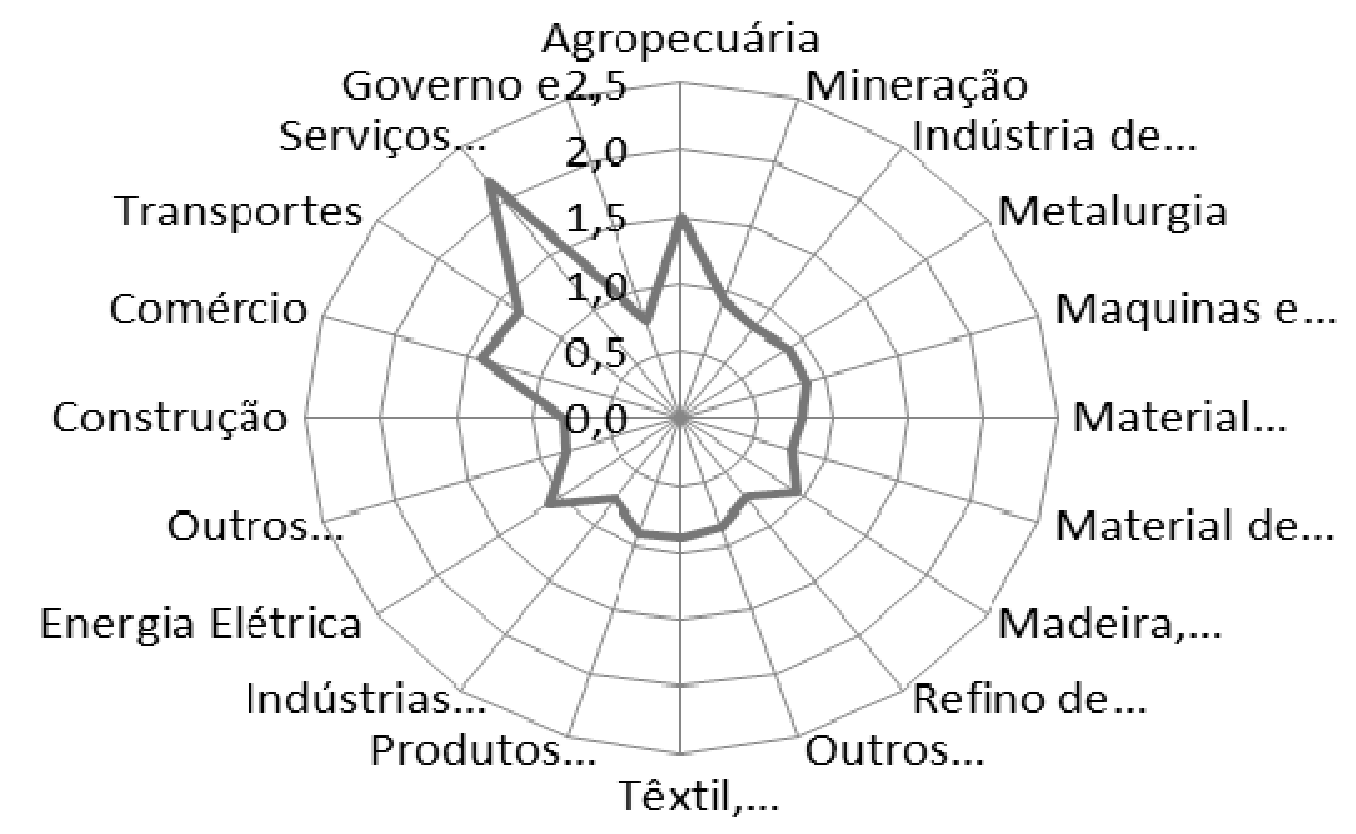

Fonte: Elaboração própria com base nas informações de Guilhoto (2008).

Apesar da maior dinâmica dessas vendas concentrarem-se no setor de serviços como observado anteriormente, a agropecuária é responsável pela composição do agronegócio que gera demandas sobre o comércio e os subsetores de transporte, armazenamento, processamento industrial e distribuição de derivados da agropecuária (CONAB, 2005; ABIGRAF, 2013).

No entanto, quando consideradas as compras intersetoriais, de acordo com o Gráfico 2, a agropecuária demonstra menor poder de resposta diante de alterações na demanda, apresentando assim poucos fornecedores de insumos, uma vez que $41 \%$ das compras do setor são provenientes da própria atividade. 
Gráfico 2 - Índices de Ligação para Trás antes da Desoneração Tributária (2004)

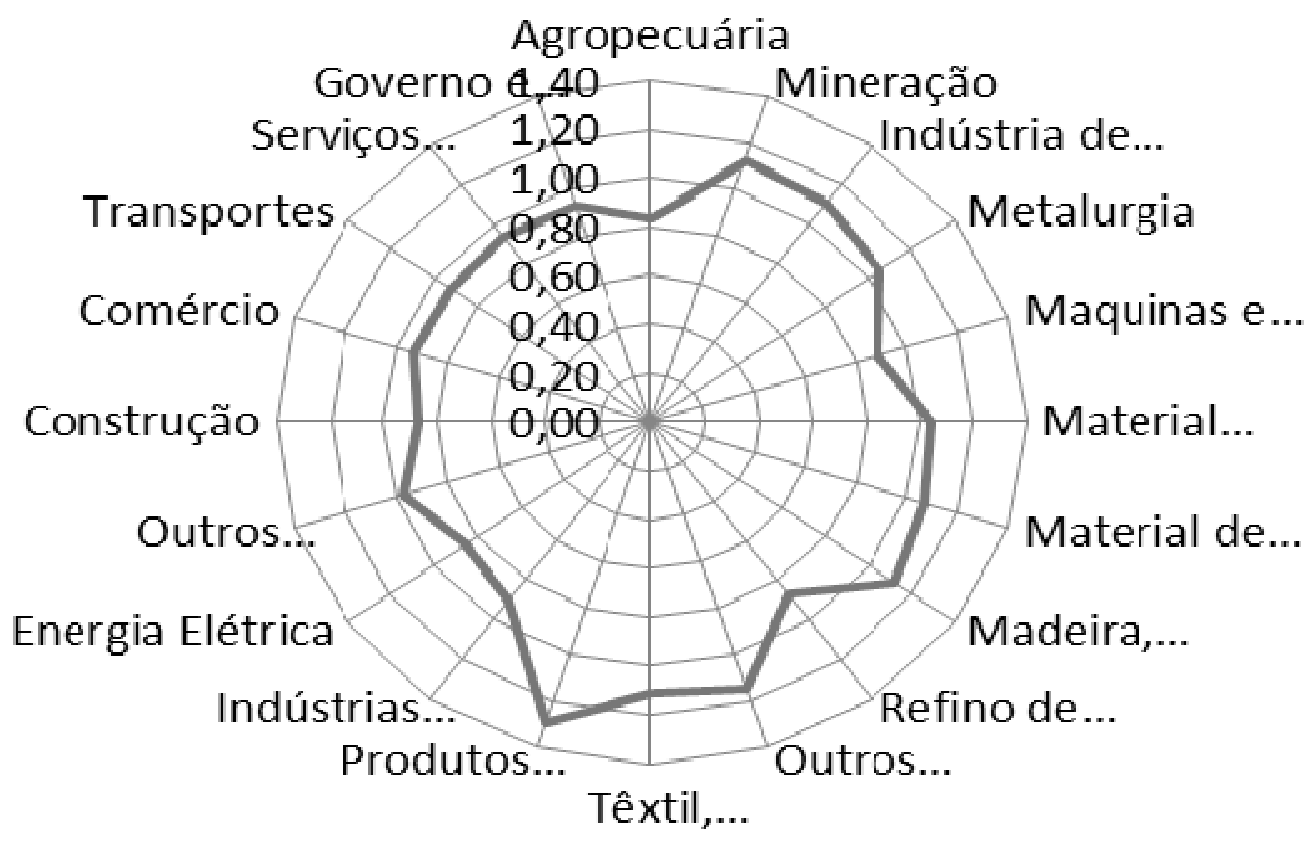

Fonte: Elaboração própria com base nas informações de Guilhoto (2008).

Diretamente, as compras e vendas do setor da agropecuária não possuem os maiores efeitos na geração de produto, emprego e renda. Mas, indiretamente, devido à mobilidade de outros setores em função desta atividade, como o comércio, geram impactos multiplicativos sobre esses efeitos que se tornam os mais significativos da economia local.

Desse modo, conforme Quadro 5, diretamente, a cada $\mathrm{R} \$ 1,00$ a mais de produto na agropecuária, a economia como um todo responde com $\mathrm{R} \$ 1,15$, mais que proporcional ao incremento no setor. De forma semelhante de interpretação, com $\mathrm{o}$ incremento de um emprego, a economia responde com 0,016 empregos. Por fim, a adição de $R$ \$1,00 na renda agropecuária, a economia reage com $R$ \$ 0,54.

A pequena representatividade na geração de empregos e rendas em relação às outras atividades deve-se, segundo o IBGE (2013), a mecanização do campo, de modo que a maior demanda por trabalhadores de forma direta e indireta se concentra em atividades relacionadas ao refino de petróleo, comércio e serviços. 
Quadro 5 - Multiplicadores produto, emprego e renda antes da desoneração tributária, 2004

\begin{tabular}{|c|c|c|c|c|}
\hline & & Produto & Emprego & Renda \\
\hline Agropecuária & 1 & 1,1555 & 0,0160 & 0,5420 \\
\hline Mineração & 2 & 1,5606 & 0,3423 & 0,4449 \\
\hline Indústria de Minerais Não Metálicos & 3 & 1,5259 & 0,0835 & 0,4528 \\
\hline Metalurgia & 4 & 1,4648 & 0,1356 & 0,3961 \\
\hline Máquinas e Equipamentos & 5 & 1,2321 & 0,2148 & 0,2712 \\
\hline Material Elétrico e Eletrônicos & 6 & 1,4512 & 0,2457 & 0,3166 \\
\hline Material de Transporte & 7 & 1,4815 & 0,2942 & 0,2473 \\
\hline Madeira, Mobiliário, Papel & 8 & 1,5519 & 0,0461 & 0,4605 \\
\hline Refino de Petróleo e Coque & 9 & 1,2070 & 4,8819 & 0,0900 \\
\hline Outros Químicos e Farmacêuticos & 10 & 1,5898 & 0,2735 & 0,4419 \\
\hline Têxtil, Vestuário, Calçados & 11 & 1,5351 & 0,0293 & 0,3779 \\
\hline Produtos Alimentícios & 12 & 1,7856 & 0,2802 & 0,2348 \\
\hline Indústrias Diversas & 13 & 1,2591 & 0,0330 & 0,3631 \\
\hline Energia Elétrica & 14 & 1,1878 & 0,8908 & 0,8495 \\
\hline $\begin{array}{l}\text { Outros serviços industriais de } \\
\text { Utilidade Pública (SIUP) }\end{array}$ & 15 & 1,3384 & 0,1296 & 0,4662 \\
\hline Construção & 16 & 1,1971 & 0,0293 & 0,5967 \\
\hline Comércio & 17 & 1,2837 & 0,0231 & 0,8276 \\
\hline Transportes & 18 & 1,2869 & 0,0433 & 0,5672 \\
\hline Serviços Privados & 19 & 1,2932 & 0,0329 & 0,8198 \\
\hline Governo e Serviços Públicos & 20 & 1,2885 & 0,0633 & 0,8289 \\
\hline
\end{tabular}

Fonte: Elaboração própria com base nas informações de Guilhoto (2008).

\section{Impactos da desoneração fiscal sobre a agropecuária}

A desoneração fiscal sobre a agropecuária é considerada fundamental para a competitividade do estado (PET'TI, 1998), uma vez que tende a reduzir os preços, de modo a aumentar a demanda e, consequentemente, o produto, cenário esse possível a partir dos aumentos nas relações intersetoriais de compra e venda. Neste contexto, dentre os tributos mais significativos, destaca-se o ICMS, que representa 80\% do imposto total que recai sobre a atividade (UFMS, 2013).

Assim, uma desoneração do ICMS em 8\% faz o preço dos produtos agropecuários se reduzir em 7,14\%, enquanto que a quantidade praticamente não se altera, uma vez que tais bens (commodities), de acordo com Varian (2006), são considerados inelásticos, em outras palavras, uma demanda pouco sensível às variações nos preços. 
Logo, em meio a essa pequena sensibilidade, a receita se reduz em 7,14\%; por outro lado, incentiva o consumo intermediário a preços básicos, em que se desconsidera a incidência tributária, gerando, portanto, efeitos multiplicadores sobre os demais setores.

Assim, após o impacto, o valor da produção total da agropecuária aumenta em $\mathrm{R} \$$ 586,50 milhões, o nível de emprego em 6,66\% e o valor adicionado em 6,64\%. Isso em decorrência das vendas intersetoriais que aumentam 29,33\% em resposta ao aumento da demanda por produtos agropecuários, provocando, inclusive, aumentos dessa venda sobre o comércio em $0,6 \%$.

Com esses resultados foi possível perceber que o impacto da desoneração sobre a agropecuária influencia positivamente as relações de venda da indústria de minerais não metálicos, outros químicos e farmacêuticos, transportes e serviços privados, setores esses importantes para viabilizarem o aumento do produto agropecuário.

Onde, em decorrência do pouco dinamismo em relação à compra de insumos, justamente, porque o estado caracteriza-se pelo fornecimento de insumo e não pela compra (CARGILL, 2013) as respostas intersetoriais da compra se reduzem.

Desse modo, diretamente, sendo os multiplicadores de emprego, renda e produto pouco significativos em relação aos demais setores, os aumentos das respostas em função da desoneração são inferiores a $1 \%$ ( $0,06 \%$ para o produto, $0,62 \%$ para o emprego e uma queda de 0,39\% no nível da renda), porém quando consideradas as influências sobre os outros setores, esses impactos são superiores em comparação à agropecuária. Onde se destacam os multiplicadores de produto, emprego e renda de outros químicos e farmacêuticos, com aumento em $67,77 \%$ e as madeiras, mobiliários e papéis em 6,45\%, como demonstrado no Quadro 6.

O aumento no percentual multiplicativo de químicos e farmacêuticos, somado a aproximadamente 3\% da indústria de minerais não metálicos e minerais em quase $0,5 \%$ podem ser justificados pelo aumento na demanda de defensivos agrícolas, adubos, fertilizantes, sais, entre outros que viabilizam a produção agropecuária. Madeira, mobiliários e papeis juntamente com outras indústrias ajudam a compor a agroindústria. Nesse sentido, a agroindústria passa a ter maiores efeitos multiplicadores.

Assim, para o escoamento do aumento produtivo tanto da agropecuária, quanto da agroindústria, quanto dos insumos, também são necessários maior quantidade de materiais de transporte, bem como combustível do setor de petróleo e coque, além do setor de transporte. Responsáveis por aproximadamente $6 \%, 3 \%$ e 0,2\% sobre produto, emprego e renda, respectivamente para esses setores.

Neste contexto, o principal setor responsável pela demanda da agropecuária, o setor de produtos alimentícios também elevou seus efeitos multiplicativos acima 
de 2\%. Com o mesmo percentual, o setor de calçados e roupas ampliou sua participação, em função da maior demanda por couro e algodão, por exemplo.

Outro destaque nestas circunstâncias se refere aos serviços e setor público, em que, devido ao aumento de receita da agropecuária e dos demais setores da economia, as contribuições tributárias detiveram aumentos multiplicadores sobre o produto em $0,13 \%$, sobre emprego $0,16 \%$ e renda $0,13 \%$, que amenizaram a redução da receita tributária refletida na desoneração sobre o setor agropecuário.

Quadro 6 - Percentual de aumento ou redução dos multiplicadores de produto, emprego e renda após o impacto (em \%)

\begin{tabular}{|c|c|c|c|c|}
\hline & & Produto & Emprego & Renda \\
\hline Agropecuária & 1 & 0,06 & 0,62 & $-0,38$ \\
\hline Mineração & 2 & 0,45 & 0,47 & 0,45 \\
\hline Indústria de Minerais Não Metálicos & 3 & 2,99 & 3,00 & 2,98 \\
\hline Metalurgia & 4 & 1,86 & 1,84 & 1,87 \\
\hline Máquinas e Equipamentos & 5 & 0,05 & 0,05 & 0,07 \\
\hline Material Elétrico e Eletrônicos & 6 & 0,61 & 0,61 & 0,60 \\
\hline Material de Transporte & 7 & 5,94 & 5,95 & 5,94 \\
\hline Madeira, Mobiliário, Papel & 8 & 6,45 & 6,29 & 6,45 \\
\hline Refino de Petróleo e Coque & 9 & 2,45 & 2,46 & 2,44 \\
\hline Outros Químicos e Farmacêuticos & 10 & 67,76 & 67,75 & 67,77 \\
\hline Têxtil, Vestuário, Calçados & 11 & 1,97 & 2,05 & 1,96 \\
\hline Produtos Alimentícios & 12 & 2,31 & 2,32 & 2,30 \\
\hline Indústrias Diversas & 13 & 1,97 & 1,82 & 1,98 \\
\hline Energia Elétrica & 14 & 0,02 & 0,02 & 0,02 \\
\hline $\begin{array}{l}\text { Outros serviços industriais de } \\
\text { Utilidade Pública (SIUP) }\end{array}$ & 15 & 0,01 & 0,08 & 0,11 \\
\hline Construção & 16 & 0,18 & 0,00 & 0,18 \\
\hline Comércio & 17 & 0,06 & 0,86 & 0,60 \\
\hline Transportes & 18 & 0,19 & 0,00 & 0,18 \\
\hline Serviços Privados & 19 & 0,12 & 0,30 & 0,12 \\
\hline Governo e Serviços Públicos & 20 & 0,13 & 0,16 & 0,13 \\
\hline
\end{tabular}

Fonte: Elaboração própria com base nas informações de Guilhoto (2008).

Logo, apesar da redução da receita tributária em $\mathrm{R} \$ 93,88$ milhões, o período estimado de recuperação foi de 0,71 anos (8,5 meses), tempo inferior ao mandato governamental e que representa a compensação total da perda tributária inicial. Além disso, tais impactos dinamizam as relações setoriais, aumentando a resposta da compra e venda para o consumo intermediário, dos multiplicadores de 
produto, renda e emprego, não apenas da agropecuária, mas de diversos setores relacionados diretamente ou indiretamente a atividade, representantes $\mathrm{da}$ promoção do desenvolvimento e crescimento econômico local.

A partir disso, conclui-se que os efeitos da desoneração seriam positivos com compensação total, em curto espaço de tempo, da redução da receita tributária atrelados ao maior dinamismo do desenvolvimento e crescimento econômico local, devido ao aumento na renda, produto e emprego.

\section{Conclusão}

A agropecuária é uma atividade fundamental para a economia estadual e nacional, ao concentrar-se entre as principais atividades relacionadas com o comércio exterior e por impulsionar o setor industrial e de serviços. Consequentemente, vinculando efeitos sobre o desenvolvimento e crescimento local, a partir, principalmente, da geração de produto, emprego e renda. No entanto, a carga tributária, principalmente, a do ICMS tende a impactar negativamente sobre essa competitividade.

De uma forma geral, mais recentemente, foi proposta a unificação do ICMS para $4 \%$, sobre todos os setores da economia e em todos os estados. Sobre a agropecuária, atualmente, o ICMS não incide sobre as exportações de bens in natura e semimanufaturados, mas ainda incide sobre manufaturados e nas operações interestaduais.

Dessa forma, ao simular o impacto dessa unificação sobre a agropecuária, obtevese, enquanto uma das confirmações nos resultados, que os produtos agropecuários são considerados inelásticos, de forma que variações nos preços implicam em pequenas respostas sobre as quantidades.

No entanto, os efeitos multiplicadores são mais significativos sobre os demais setores envolvidos diretamente e indiretamente na agropecuária, que resultaram no aumento de produto, emprego e renda. Isto em decorrência do setor ser considerado chave no estado de Mato Grosso do Sul. Mesmo considerando uma redução na receita tributária, o tempo de recuperação seria inferior a um ano, o que mostra a eficiência dessa política durante o mandato governamental de quatro anos.

Diante desses impactos, o dinamismo de vendas para o consumo intermediário decorrente dos aumentos do produto, emprego e renda se eleva; por outro lado, as compras se reduzem por caracterizar-se o setor como fornecedor de insumos.

Portanto, diante desses resultados sobre a tributação e geração de produto, emprego e renda, a desoneração do ICMS para 4\% é viável. Recomendando-se ao setor público a implementação dessa política de forma imediata, na busca pela 
competitividade do setor e impactos positivos sobre o desenvolvimento e crescimento econômico do estado.

Cabem a trabalhos futuros pesquisas de campo que impliquem na realidade do estado de Mato Grosso do Sul, com desencadeamento e uma maior desagregação da atividade agropecuária, além da contemplação dos demais setores da economia.

\section{Referências}

ABIGRAF. Associação Brasileira da Indústria Gráfica. Mato Grosso do Sul. Disponível em: <http://www.abigraf.org.br/index.php/abigrafs-regionais/matogrosso-do-sul>. Acesso em: 6 jun. 2013.

ARAÚJO, Herbert. V. Impactos da desoneração fiscal na receita tributária, emprego e renda e cálculo do payback tributário. Monografia (graduação), 2007. XII Prêmio do Tesouro Nacional. Brasília: 2007.

BACHA, Carlos J. C. Economia e política agrícola no Brasil. São Paulo: Atlas, 2004.

BALDWIN, Robert. E. Desenvolvimento e crescimento econômico. São Paulo: Pioneira, 1979.

BRASIL. Ações Federais - Mato Grosso do Sul - 1995/2002. 2002. Disponível em: <http://www.abrasil.gov.br/estados/pdf/ms.pdf>. Acesso em: 22 abr. 2013.

. Lei Complementar Anterior $\mathbf{n}^{\circ} 87$ de 1996. Dispõe sobre o imposto dos Estados e do Distrito Federal sobre operações relativas à circulação de mercadorias e sobre prestações de serviços de transporte interestadual e intermunicipal e de comunicação, e dá outras providências. (Lei Kandir). Brasília: 1996. Disponível em: <http://www.planalto.gov.br/ccivil_03/ leis/lcp/lcp87.htm>. Acesso em: 28 out. 2013.

Lei $N^{\circ}$ 12.512, de 14 de Outubro de 2011. Institui o Programa de Apoio à Conservação Ambiental e o Programa de Fomento às Atividades Produtivas Rurais; altera as Leis $\mathrm{N}^{\mathrm{0}}$ 10.696, de 2 de julho de 2003, 10.836, de 9 de janeiro de 2004, e 11.326, de 24 de julho de 2006. Diário Oficial [da] República Federativa do Brasil. Brasília, DF, 14 out. 2011.

. Medida Provisória $n^{\circ}$ 599, de 27 de dezembro de 2012. Dispõe sobre a prestação de auxílio financeiro pela União aos Estados, ao Distrito Federal e aos Municípios, com o objetivo de compensar perdas de arrecadação decorrentes da 
redução das alíquotas nas operações e prestações interestaduais relativas ao Imposto sobre Operações relativas à Circulação de Mercadorias e sobre Prestações de Serviços de Transporte Interestadual e Intermunicipal e de Comunicação - ICMS, institui o Fundo de Desenvolvimento Regional e dá outras providências. Brasília: 2012. Disponível em: <http://www.planalto.gov.br/ ccivil_03/_Ato2011-2014/2012/Mpv/599.htm>. Acesso em: 22 fev. 2013.

BUILDER. Demanda de Produtos Agrícolas. [2004?]. Disponível em: <http://materiais.tripod.com/sitebuildercontent/sitebuilderfiles/demanda.pdf $>$ Acesso em: 04 jun. 2013.

CARGILL. Agrícola. 2013. Disponível em: <http://www.cargill.com.br/pt/ produtos-servicos/agricola/index.jsp>. Acesso em: 13 out. 2013.

CLEMENTE, Ademir; HIGACHI, Hermes Y. Economia e desenvolvimento regional. São Paulo: Atlas, 2000.

CONAB. Companhia Nacional de Abastecimento. Armazenagem agrícola no Brasil. 2005. Disponível em: <http://www.conab.gov.br/OlalaCMS/uploads/ arquivos/7420aabad201bf8d9838f446e17c1ed5..pdf $>$ Acesso em: 20 set. 2013.

CORREAA, Lucia. S. A Construção de um Novo Estado - Mato Grosso do Sul. 2011. Disponível em: <http://www.ihgms.com.br/index.php?option= com_content\&view =article\&id $=134:$ a-construcao-de-um-novo-estado\&catid $=5$ 3:lucia-salsa-correa\&Itemid=137>. Acesso em: 24 abr. 2013.

CRUZ, Valdo; OMS, Carolina. Desoneração da cesta básica não dever ser integral, dizem analistas. Brasília, DF: Folha de S. Paulo, 2013. Disponível em: <http://app.folha.com/m/noticia/223403> Acesso em: 02 jun. 2013.

DÜRR, Jochen; COSTA, Francisco A. de. Cadeias produtivas de base agrária e desenvolvimento regional: o caso da Região do Baixo Tocantins. In: Revista Amazônia: CI \& Desenv. Belém: v. 3, nº 6, 2008, 38 p.

FEIJÓ, Carmen A.; RAMOS, Roberto L. O. Contabilidade social: a nova referência das contas nacionais do Brasil. 3. ed. rev. e atual. Rio de Janeiro: Elsevier: Campus, 2004.

GUILHOTO, Joaquim. J. M. Matriz inter-regional de Insumo-Produto para o Brasil 2004 - 20 Setores - MS e RBR. São Paulo: NEREUS, 2008.

HOFFMANN, Rodolfo. Elasticidades-Renda das despesas e do consumo de alimentos no Brasil em 2002-2003. S.L.: 2007. 
IBGE. IBGE publica Matriz de Insumo-Produto para os anos 2000 e 2005. 2008a. Disponível em: <http://www.ibge.gov.br/home/presidencia/ noticias/matrizinsumo_produto.shtm>. Acesso em: 14 mai. 2013.

Comércio Agropecuário por vias internas, consumo intermediário e valor bruto da produção agropecuária. Planilhas. Campo Grande - MS: SEMAC, 2008b.

- Mecanização Elimina Postos de Trabalho no Campo. 2013. Disponível em: <http://economia.terra.com.br/ibge-mecanizacao-eliminapostos-de-trabalho-no-campo,e70882ddb7f61410VgnCLD200000bbcceb0aRCR D.html>. Acesso em: 30 set. 2013.

. PIB Estadual. 2010. Disponível em: <http://www.ibge.gov.br>. Acesso em: 16 dez. 2012.

LAKATOS, Eva M.; MARCONI, Mariana A. de. Fundamentos de metodologia científica. 6. ed. São Paulo: Atlas, 2008.

LEONTIEF, Wassily. A economia do insumo-produto. São Paulo: Abril Cultural, 1983.

LIMA, Djalba. Reforma transfere peso da cobrança do ICMS ao destino das mercadorias. Jornal do Senado, 06/05/2013. Disponível em: <http://www12.senado.gov.br/jornal/edicoes/2013/05/06/reforma-transferepeso-da-cobranca-do-icms-ao-destino-das-mercadorias >. Acesso em: 7 mai. 2013.

MAIA NETO, Adalberto A.; BÊRNI, Duílio A. de. Técnicas de pesquisa em economia: Transformando Curiosidade em Conhecimento. São Paulo: Saraiva, 2002.

MUSGRAVE, Richard A.; MUSGRAVE, Peggy B. Finanças públicas: teoria e prática. [s.1.]: Campus, 1980, 673 p.

PEREIRA, Janaína. S. A importância do complexo soja nas macrorregiões brasileiras: uma análise utilizando matrizes insumo-produto inter-regionais. 2010. 72 p. Monografia (Graduação em Ciências Econômicas) - Universidade Federal de Viçosa, Viçosa - MG, 2010.

PETTI, Regina H. V. ICMS e agropecuária: conquistas recentes. São Paulo: IEA e SAAESP, 1998. 
PINAZZA, Luiz A. Cadeia produtiva da soja. Brasília: MAPA, SPA e IICA, v. 2, 2007.

RODRIGUES, Adyr A. B. Turismo e desenvolvimento local. 2. ed. São Paulo: Hucitec, 2000.

SEFAZ. Lei de Responsabilidade Fiscal - 2003/2010. 2013. Disponível em: <http://arq.sefaz.ms.gov.br/. Acesso em: 3 jun. 2013.

SEMAC/MS e CONAB/IBGE. Série histórica PIB Mato Grosso do Sul 19952010. Mato Grosso do sul: SEMAC, 2010.

SOUZA, Nali de. Desenvolvimento econômico. 5. ed. São Paulo: Atlas, 2009.

SOUZA, André R. P.; STICCA, Ralph. M. Aspectos de Tributação da Produção, Industrialização e Comercialização Agropecuária no Brasil. In: Renato Buranello, André Ricardo Passos de Souza e Ecio Perin Junior (Coord.). Direito do Agronegócio: Mercado, Regulação, Tributação e Meio Ambiente. 1. ed. (IBRADEMP). São Paulo: Quartier Latin, p. 740-789, 2011.

TALAMINI, Edson; PEDROZO, Eugênio A. Matriz de Insumo-Produto (MIP) e alguns indicadores para a gestão e planejamento de propriedades rurais: uma aplicação prática. In: Revista teoria e evidência econômica, Passo Fundo, v. 12, n. 23, p. 25-43, 2004.

UFMS. Universidade Federal de Mato Grosso do Sul. Relatório de pesquisa elaboração da TRU e construção da Matriz Insumo-Produto 2008. Campo Grande, MS: Universidade Federal de Mato Grosso do Sul, 2013.

UNIDERP. Universidade para o Desenvolvimento do Estado e Região do Pantanal. Inflação. Mato Grosso do Sul: UNIDERP, 2004.

VARIAN, Hal R. Microeconomia: princípios básicos - uma abordagem moderna. Rio de Janeiro: Elsevier, 2006. 
Endereço para correspondência:

Mayra Batista Bitencourt Fagundes - bitencourtmayra@gmail.com

Cidade Universitária

79070-900 Campo Grande/MS, Brasil

Daniela Teixeira Dias - danielateixeiradias@hotmail.com

Cidade Universitária

79070-900 Campo Grande/MS, Brasil

Daniel Massen Frainer - danielfrainer@gmail.com

BR 463, Km 4,5 Cidade Universitária

79900-000 Ponta Porã/MS, Brasil

Cícero Antônio Oliveira Tredezinin - cicero.tredezini@gmail.com

Avenida Universitária, s/n.

79059-900 Campo Grande/MS, Brasil

Leonardo Francisco Figueiredo Neto - lffneto@gmail.com

Av. Senador Filinto Müller, Unidade 10

79070-900 Campo Grande/MS, Brasil

144 | Revista Brasileira de Desenvolvimento Regional, Blumenau, 2 (1), P. 119-144, outono de 2014 